\title{
Note de recherche
}

\section{Création d'un hybride trispécifique de cotonnier résistant au nématode réniforme}

\author{
Olivier Konan N Guessan ${ }^{1}$ \\ Jean-Pierre Baudoin ${ }^{1}$ \\ Angélique D'Hont ${ }^{2}$ \\ Guy Mergeai ${ }^{1}$ \\ ${ }^{1}$ Unité de phytotechnie tropicale \\ et d'horticulture, \\ Faculté universitaire \\ des sciences agronomiques, \\ 2, passages des Déportés, \\ B-5030 Gembloux, Belgique \\ $<$ konan.n@fsagx.ac.be> \\ $<$ baudoin.jp@fsagx.ac.be> \\ $<$ mergeai.g@fsagx.ac.be> \\ ${ }^{2}$ Centre de coopération internationale \\ en recherche agronomique \\ pour le développement (Cirad), \\ Unité mixte de recherche (UMR) 1096 PIA, \\ TA 40/03, \\ F-34398 Montpellier cedex 5 \\ France \\ <angelique.dhont@cirad.fr>
}

\begin{abstract}
Résumé
Le nématode réniforme Rotylenchulus reniformis Linford et Oliveira est en passe de devenir le nématode le plus redoutable de la culture cotonnière en Amérique du Nord et du Sud. Dans le but d'introgresser chez la principale espèce de cotonnier cultivé Gossypium hirsutum L. la résistance à ce parasite à partir de l'espèce diploïde sauvage G. longicalyx, l'hybride trispécifique G. hirsutum x G. thurberi x G. longicalyx a été créé selon la méthode pseudophylétique d'introgression. La triple hybridité du matériel produit a été mise en évidence au moyen de marqueurs microsatellites et confirmée par le comptage des chromosomes. Trois plantes hybrides évaluées en conditions d'inoculation contrôlée ont révélé un niveau de résistance à $R$. reniformis élevé et équivalent à celui de l'espèce diploïde donneuse.
\end{abstract}

Mots clés : coton ; hybridation interspécifique ; nématode des plantes ; résistance génétique.

Thèmes : productions végétales; méthodes et outils.

\section{Abstract \\ Creation of a cotton trispecific hybrid resistant to the reniform nematode}

The reniform nematode Rotylenchulus reniformis Linford and Oliveira is becoming one of the most important constraints of cotton production in North and South America. In order to introgress into the main cultivated cotton species Gossypium hirsutum L. the resistance to this parasite from the wild cotton diploid species G. longicalyx, the G. hirsutum $\mathrm{x}$ G. thurberi $\mathrm{x}$ G. longicalyx trispecific hybrid has been created according to the pseudophyletic method of introgression. The triple species character of the hybrid produced has been proved by using simple sequence repeat (SSR) markers and confirmed by chromosome counting. Three hybrid plants evaluated in controlled conditions of inoculation revealed a high level of resistance to $R$. reniformis equivalent to that of the diploid donor species.

Key words: cotton; genetic resistance; interspecific hybridization; plant nematodes.

Subjects: vegetal productions; tools and methods. e nématode réniforme Rotylenchulus reniformis Linford et Oliveira est un ver microscopique semiendoparasite sédentaire des régions chaudes dont la femelle attaque les racines de ses plantes hôtes. Il est aujourd'hui en passe de devenir le nématode le plus redoutable de la culture cotonnière en Amérique du Nord et du Sud (Westphal et Scott, 2005). Les pertes quantitatives de récolte dues à ce nématode vont de 30 à
$60 \%$ selon les niveaux d'infestation et les conditions météorologiques (Yik et Birchfield, 1984). Parmi les moyens de lutte envisageables pour faire face à ce parasite (rotation des cultures, travail du sol, gestion des mauvaises herbes, utilisation de nématicides), le recours à des variétés de cotonnier résistantes est potentiellement le moyen le plus efficace, le plus économique et le plus respectueux de l'environnement. À ce jour, 
aucune source de résistance à $R$. reniformis n'a été identifiée au sein du réservoir génétique primaire de l'espèce G. birsutum (Westphal et Scott, 2005) et seuls un petit nombre de cotonniers diploïdes sauvages semblent pouvoir être utilisés comme espèces donneuses de ce caractère. Parmi ces espèces, G. longicalyx est celle qui présente le plus haut niveau de résistance au nématode réniforme (Yik et Birchfield, 1984). Plusieurs équipes de recherche tentent aujourd'hui aux ÉtatsUnis d'utiliser G. longicalyx pour introgresser la résistance à $R$. reniformis chez G. birsutum (Robinson et al., 2005). Leurs travaux ont abouti à la création des hybrides triples "HLA " [ $G$. hirsutum x G. longicalyx) ${ }^{2}$ x G.armourianum] et "HHL" $\left[(G \text {. hirsutum x G. herbaceum })^{2} \mathrm{x}\right.$ $G$. longicalyx]. Nous présentons ici l'état d'avancement de nos recherches concernant le développement par hybridation interspécifique de cotonniers génétiquement équilibrés, agronomiquement compétitifs et résistants à $R$. reniformis.

\section{Matériel et méthode}

La méthode pseudophylétique d'introgression (Mergeai, 2006) a été mise en œuvre pour produire l'hybride trispécifique G. hirsutum x G. thurberi x G. longicalyx (figure 1). Cette méthode, tout comme la méthode paraphylétique, vise l'introgression de gènes intéressants chez le cotonnier cultivé via la création d'un hybride trispécifique qui est un tétraploïde synthétique (Mergeai, 2006). La méthode paraphylétique est cependant plus difficile à mettre en œuvre car les croisements directs entre diploïdes qui constituent sa première étape sont plus difficiles à réaliser que les hybridations entre tétraploïdes et diploïdes.

Six marqueurs microsatellites montrant un polymorphisme simple à interpréter chez les trois espèces de cotonnier constitutives de l'hybride HTL ont été utilisés pour vérifier le caractère hybride du matériel produit. Les protocoles suivis pour réaliser l'extraction de l'ADN et les analyses SSR étaient ceux de Vroh Bi et al. (1996) et de Liu et al. (2000).

Le nombre de chromosomes des hybrides créés a été déterminé par analyse cytogénétique moléculaire sur des cellules de pointes de racines en division active selon la méthode décrite par D'Hont et al. (1995).

L'évaluation de la résistance à $R$. reniformis s'est faite par la mesure du degré de

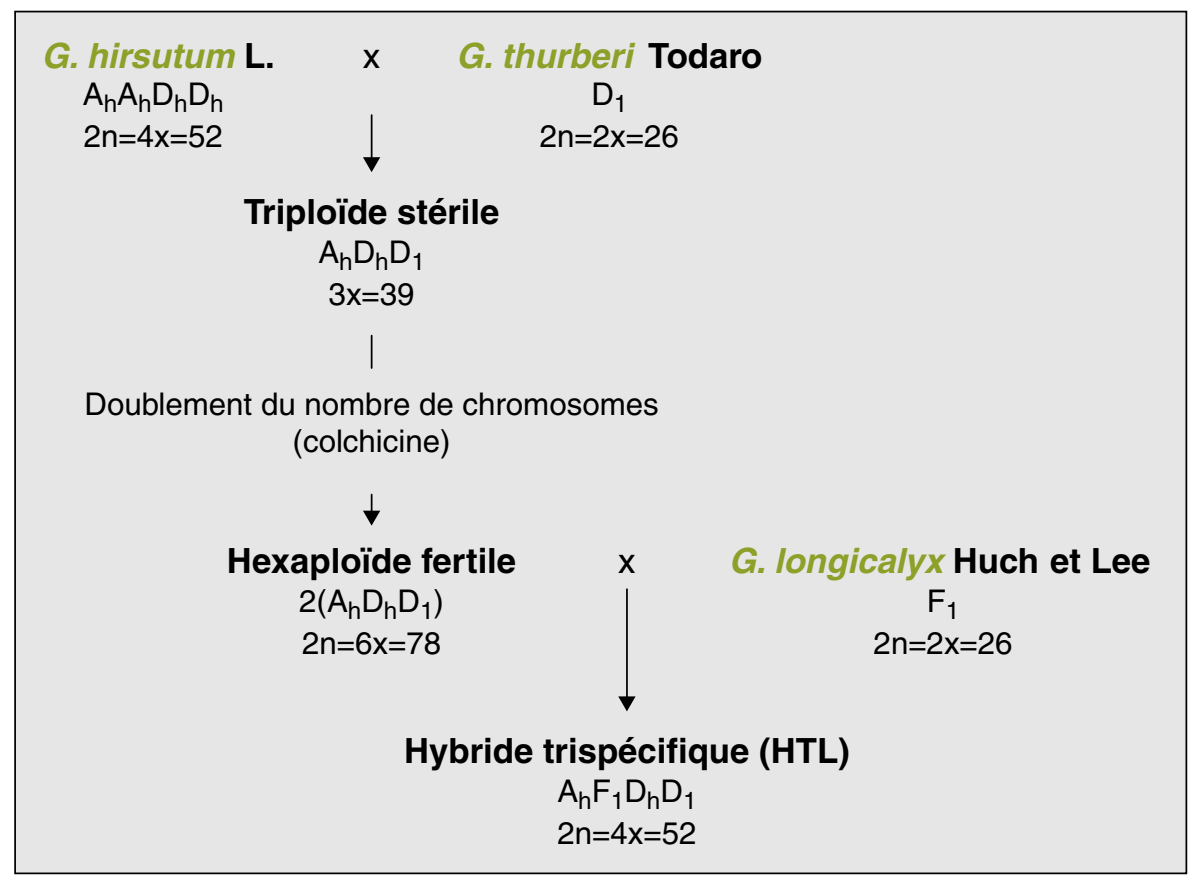

Figure 1. Schéma de création de I'hybride HTL (G. hirsutum x G. thurberix G. longicalyx) par la méthode pseudophylétique d'introgression.

Figure 1. Development scheme of the HTL hybrid (G. hirsutum $x$ G. thurberi $\times$ G. longicalyx) by the pseudophyletic method of introgression.

reproduction du nématode sur la plante en comptant le nombre d'œufs produits à la fin du cycle de reproduction du parasite et en rapportant ce nombre à la masse de racines (matière fraîche) produites par plante. L'inoculation a été faite sur des plantules de 30 jours par injection d'une suspension de 6000 œufs dans le substrat de culture; après 60 jours d'infestation, les œufs ont été extraits par centrifugation-flottation et comptés sous binoculaire dans des boîtes de Petri divisées en bandes parallèles. Une fois le nombre d'œufs déterminé, le degré de résistance de la plante a été estimé selon l'échelle de sensibilité relative proposée par Yik et al. (1984) en comparant le nombre d'œufs isolés par gramme de racine chez les différents génotypes testés et chez le témoin sensible G. hirsutum.

\section{Résultats}

Dix graines ont été obtenues sur un total de 34 croisements réalisés entre l'hexaploïde G. hirsutum x G. thurberi et G. longicalyx. Neuf de ces graines ont donné des plantes viables.

Les analyses réalisées au moyen de six marqueurs microsatellites ont montré, d'une part, un profil de migration des bandes identique chez les 9 plantes hybrides et, d'autre part, une additivité allélique par la présence commune chez ces plantes des allèles spécifiques de chacune des trois espèces constitutives (figure 2).

Les analyses cytogénétiques ont révélé des formules caryologiques conformes aux résultats attendus : 52 chromosomes pour G. hirsutum et les hybrides HLT, 26 chromosomes pour G. thurberi et G. longicalyx, et 78 chromosomes pour l'hexaploïde G. hirsutum x $G$. thurberi.

Les résultats obtenus à l'aide des marqueurs microsatellites et par cytogénétique moléculaire confirment la réussite du croisement interspécifique et le caractère trispécifique des plantes hybrides obtenues. Celles-ci sont de ce fait des tétraploïdes synthétiques et peuvent être désignées par l'abréviation HTL ( $\mathrm{H}=$ hirsutum; $\mathrm{T}=$ thurber $; \mathrm{L}=$ longicaly $x$ ).

Trois plantes HTL sur les neuf obtenues ont été testées pour leur résistance au nématode réniforme tandis que les autres ont été utilisées directement pour être croisées par différentes variétés de $G$. hirsutum. Les trois plantes testées se sont révélées aussi résistantes vis-à-vis de $R$. reniformis que l'espèce donneuse du caractère G.longicalyx. Nous y avons 


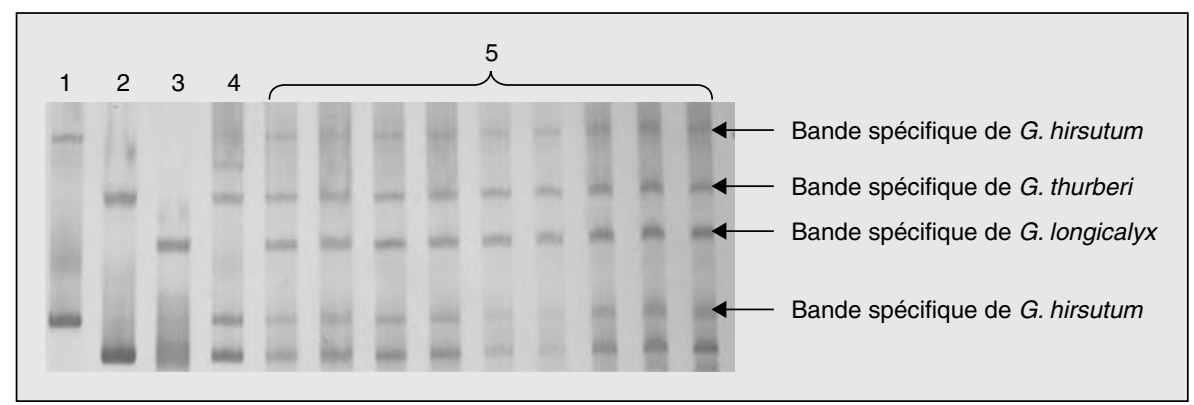

Figure 2. Profil de migration microsatellite de l'amorce BNL 4030 montrant le caractère trispécifique des hybrides HTL.

Figure 2. SSR electrophoretic profile of the primer BNL 4030 showing the triple species character of the HTL hybrids.

1) G. hirsutum ; 2) G. thurberi ; 3) G. longicalyx ; 4) hexaploïde (G. hirsutum x G. thurberi) ; 5) neuf hybrides (G. hirsutum $\times$ G. thurberix G. longicalyx).

compté respectivement: 5,$80 ; 4,09$ et 1,77 œufs par gramme de racine, soit en moyenne moins de $2,5 \%$ du nombre observé chez le témoin sensible G. birsutum (tableau 1).

\section{Conclusion}

Nous pouvons conclure des résultats obtenus jusqu'à présent que les hybri-

\section{Tableau 1. Résultats de l'évaluation de la résistance à $\boldsymbol{R}$. reniformis de trois hybrides HTL et de leurs parents.}

Table 1. Results of the evaluation of the resistance to $R$. reniformis of three HTL hybrids and their parents.

\begin{tabular}{lcccc}
\hline Génotypes & $\begin{array}{c}\text { Nb moyen } \\
\text { d'oeufs } \\
\text { produits } \\
\text { par plante }\end{array}$ & $\begin{array}{c}\text { Masse } \\
\text { racinaire } \\
\text { moyenne } \\
\text { par plante (g) }\end{array}$ & $\begin{array}{c}\text { Nb moyen } \\
\text { d'œufs par g } \\
\text { de racine }\end{array}$ & $\begin{array}{c}\text { Degré de } \\
\text { résistance }\end{array}$ \\
\hline $\begin{array}{l}\text { G. hirsutum } \\
\text { (cv C2) }\end{array}$ & 53894 & 106,54 & 506 & Sensible \\
(2 plantes) & 7407 & 23,91 & 310 & Sensible \\
$\begin{array}{l}\text { G. thurberi } \\
\text { G. longicalyx } \\
\text { (2 plantes) }\end{array}$ & 253 & 21,20 & 12 & Très résistant \\
$\begin{array}{l}\text { HTL } \\
\text { (3 plantes) }\end{array}$ & 314 & 79,69 & 4 & Très résistant \\
\hline
\end{tabular}

des HTL créés à Gembloux constituent un matériel de haute valeur pour introgresser la résistance au nématode réniforme chez la principale espèce de cotonnier cultivé et que la résistance à ce nématode apportée par G. longicalyx doit être un caractère dominant car elle s'exprime à un très haut niveau chez des hybrides tétraploïdes totalement hétérozygotes. Les plantes hybrides produites font actuellement l'objet de croisements par différentes variétés de $G$. hirsutum dans le but de produire des cotonniers génétiquement équilibrés, agronomiquement compétitifs et résistants à $R$. reniformis.

\section{Références}

D'Hont A, Rao PS, Feldmann P, Grivet L. Identification and characterisation of sugarcane intergeneric hybrids, Saccharum officinarum $x$ Erianthus arundinaceus, with molecular markers and DNA in situ hybridisation. Theor Appl Genet $1995 ; 91$ : 320-6.

Liu S, Cantrell RG, McCarty JC, Stewart J. Simple sequence repeat-based assessment of genetic diversity in cotton race stock accessions. Crop Sci $2000 ; 40$ : 1459-69.

Mergeai G. Introgressions interspécifiques chez le cotonnier. Cah Agric 2006 ; 15 : 135-43.

Robinson AF, Bell AA, Stelly DM, Dighe ND, Menz MA. Introgression of Resistance to Roty lenchulus reniformis into Gossypium hirsu tum from G. longicalyx. J Nematol $2005 ; 37$ 391.

Vro H, Bi I, Chandelier A, Mergeai G, Du, Jardin P. Improved RAPD amplification of recalcitrant plant DNA by the use of activated charcoal during DNA extraction. Plant Breed 1996 $115: 205-6$.

Westphal A, Scott AW. Implementation of soybean in cotton cropping sequences for management of reniform nematode in South Texas. Crop Sci $2005 ; 45$ : 233-9.

Yik CP, Birchfield W. Resistant germplasm in Gossypium species and related plants to Roty lenchulus reniformis. J Nematol 1984; 16 : 146-53. 